\title{
Strong-coupling analysis of large bipolarons in two and three dimensions
}

\author{
G. Verbist, M. A. Smondyrev, ${ }^{*}$ F. M. Peeters, and J. T. Devreese ${ }^{\dagger}$ \\ Universiteit Antwerpen (UIA), Departement Natuurkunde, B-2610 Antwerpen, Belgium
}

(Received 13 May 1991)

\begin{abstract}
In the limit of strong electron-phonon coupling, we use either a Pekar-type or an oscillator wave function for the center-of-mass coordinate and either a Coulomb or an oscillator wave function for the relative coordinate, and are able to reproduce all the results from the literature for the large-bipolaron binding energy. Lower bounds are constructed for the critical ratio $\eta_{c}$ of dielectric constants below which bipolarons can exist. It is found that, in the strong-coupling limit, the stability region for bipolaron formation is much larger in two dimensions (2D) than in 3D. We introduce a model that combines the averaging of the relative coordinate over the asymptotically best wave function with a path-integral treatment of the center-of-mass motion. The stability region for bipolaron formation is increased compared with the full path-integral treatment at large values of the coupling constant $\alpha$. The critical values are $\alpha_{c} \approx 9.3$ in $3 \mathrm{D}$ and $\alpha_{c} \approx 4.5$ in $2 \mathrm{D}$. Phase diagrams for the presented models are also obtained in both $2 \mathrm{D}$ and $3 \mathrm{D}$.
\end{abstract}

\section{INTRODUCTION}

Two identical charged particles (electrons) being placed in a polar or ionic crystal will interact with the lattice vibrations that will result in attractive forces between them. Under certain conditions a bound state can exist, and it will consist of two electrons and a common cloud of (virtual) phonons. Such a quasiparticle is referred to as a bipolaron.

Recently, there has been renewed interest in the bipolaron problem, which was triggered by the possibility of $b i$ polaronic superconductivity in the high-temperature superconductors. Bipolarons act as charged bosons that could undergo Bose-Einstein condensation. For large bipolarons such a mechanism was discussed by Emin and Hillary. ${ }^{1}$ This mechanism is based on a Bose-Einstein condensation of the bipolarons as discussed by Shafroth. ${ }^{2}$ Recently, some questions on Shafroth's results have been raised by Friedberg, Lee, and Ren. ${ }^{3}$ These remarks, however, have no bearing on the present work, since we study the stability region of one bipolaron, not the manyparticle aspects of the bipolaron gas.

The applicability of the bipolaron theories of superconductivity depends upon the existence of bipolarons in the superconducting materials. Here we will study characteristics of bipolarons such as the self-energy, effective mass, radius and average number of virtual phonons. A large number of papers ${ }^{4-14}$ have already been devoted to this problem. To the best of the authors' knowledge, Moskalenko ${ }^{4}$ was the first to study the bipolaron problem in the strong-coupling limit. In later work wo $^{5-14}$ it was concluded that bipolarons can exist at some critical values of the electron-phonon coupling constant and Coulomb repulsion coefficient. In order that a bipolaron can exist, the repulsive forces should be weak enough, while the electron-phonon interaction has to be sufficiently strong to overcome that repulsion.

To calculate the critical values of the coupling con- stant, different authors used different versions of variational upper estimates for the bipolaron energy. Some of them differ by an order of magnitude. The aim of the present paper is to present a comparative study of the different methods for the calculation of the bipolaron binding energy. In doing so we will also calculate other bipolaron characteristics such as the effective mass, radius, and average number of virtual phonons in the bipolaron cloud.

The paper is organized as follows. In Sec. II we consider various versions of variational upper estimates to the bipolaron energy in the strong-coupling limit. This will allow us to determine the best estimate to the bipolaron binding energy. The latter is used in Sec. III to derive approximate expressions for the bipolaron spinsinglet state. In Sec. IV we discuss the results obtained and compare them with those of other authors. Our conclusions are presented in Sec. V.

\section{VARIATIONAL ESTIMATES FOR THE BIPOLARON ENERGY}

A system of two electrons interacting with a phonon field is described by the Fröhlich Hamiltonian

$$
\begin{aligned}
H= & \sum_{j=1,2}\left(\frac{\mathbf{p}_{j}^{2}}{2 m}+\sum_{\mathbf{k}}\left(V_{\mathbf{k}} a_{\mathbf{k}} e^{i \mathbf{k} \cdot \mathbf{r}_{j}}+V_{\mathbf{k}}^{*} a_{\mathbf{k}}^{\dagger} e^{-i \mathbf{k} \cdot \mathbf{r}_{j}}\right)\right) \\
& +\sum_{\mathbf{k}} \hbar \omega_{\mathbf{k}} a_{\mathbf{k}}^{\dagger} a_{\mathbf{k}}+U\left(\left|\mathbf{r}_{1}-\mathbf{r}_{2}\right|\right),
\end{aligned}
$$

where $\mathbf{r}_{j}\left(\mathbf{p}_{j}\right)$ is the position (momentum) operator of the $j$ th electron $(j=1,2), m$ is the electron band mass, and $U(r)=U / r$ is the Coulomb interaction potential with strength $U$. The technique to determine the ground state of the Hamiltonian [Eq. (1)] is quite general, but we shall concentrate on the three-dimensional bipolaron in the case of longitudinal-optical (LO) phonons, which are assumed to be dispersionless, $\omega_{\mathbf{k}}=\omega_{\mathrm{LO}}$, with the following form for the interaction coefficients: 


$$
V_{\mathbf{k}}=-i \hbar \omega_{\mathrm{LO}}\left[\frac{4 \pi \alpha}{k^{2} V}\left[\frac{\hbar}{2 m \omega_{\mathrm{LO}}}\right]^{1 / 2}\right]^{1 / 2},
$$

where $V$ is the crystal volume and $\alpha$ is the dimensionless electron-phonon coupling constant,

$$
\alpha=\frac{1}{\hbar \omega_{\mathrm{LO}}} \frac{e^{2}}{2}\left[\frac{1}{\epsilon_{\infty}}-\frac{1}{\epsilon_{0}}\right]\left(\frac{2 m \omega_{\mathrm{LO}}}{\hbar}\right)^{1 / 2},
$$

where $\epsilon_{\infty}\left(\epsilon_{0}\right)$ is the high-frequency (static) dielectric constant. The nonscreened Coulomb repulsion strength is given by $U=e^{2} / \epsilon_{\infty}$. For convenience, we shall use a system of units in which $\hbar=m=\omega_{\mathrm{LO}}=1$. One can easily verify that in these units the following inequality holds:

$$
U \geq \sqrt{2} \alpha .
$$

Since the bipolaron is a composite particle, we introduce a center-of-mass coordinate $\mathbf{R}=\frac{1}{2}\left(\mathbf{r}_{1}+\mathbf{r}_{2}\right)$ $\left[\mathbf{P}=\mathbf{p}_{1}+\mathbf{p}_{2}\right]$ and a relative coordinate $\mathbf{r}=\mathbf{r}_{1}-\mathbf{r}_{2}$ $\left[\mathbf{p}=\left(\mathbf{p}_{1}-\mathbf{p}_{2}\right) / 2\right]$ for the electron pair. In terms of these coordinates, the Hamiltonian (1) can be rewritten as

$$
\begin{aligned}
H= & \frac{\mathbf{P}^{2}}{4}+\mathbf{p}^{2}+2 \sum_{\mathbf{k}} \cos \left(\frac{\mathbf{k} \cdot \mathbf{r}}{2}\right)\left(V_{\mathbf{k}} a_{\mathbf{k}} e^{i \mathbf{k} \cdot \mathbf{R}}+\text { H.c. }\right) \\
& +\sum_{\mathbf{k}} a_{\mathbf{k}}^{\dagger} a_{\mathbf{k}}+\frac{U}{r} .
\end{aligned}
$$

To obtain variational upper estimates of the bipolaron self-energy, we average the Hamiltonian (5) over the product-wave-function ansatz

$$
\Psi\left(\mathbf{r}_{1}, \mathbf{r}_{2}\right)=\Phi(\mathbf{R}) \varphi(\mathbf{r})
$$

and obtain the effective Hamiltonian

$$
\mathscr{H}=E_{R}+E_{r}+\sum_{\mathbf{k}} a_{\mathbf{k}}^{\dagger} a_{\mathbf{k}}+\sum_{\mathbf{k}}\left(A_{\mathbf{k}} a_{\mathbf{k}}+\text { H.c. }\right),
$$

with the ground-state energy

$$
E=E_{R}+E_{r}-\sum_{\mathbf{k}}\left|A_{\mathbf{k}}\right|^{2}
$$

The following notations were introduced:

$$
\begin{aligned}
& E_{R}=\left\langle\left\langle\frac{\mathbf{P}^{2}}{4}\right\rangle, E_{r}=\left\langle\mathbf{p}^{2}+\frac{U}{r}\right\rangle,\right. \\
& A_{\mathbf{k}}=2 V_{\mathbf{k}}\left\langle\left\langle e^{i \mathbf{k} \cdot \mathbf{R}}\right\rangle\right\rangle\left\langle\cos \left(\frac{\mathbf{k} \cdot \mathbf{r}}{2}\right)\right\rangle,
\end{aligned}
$$

where $\langle\cdots\rangle[\langle\langle\rangle\rangle]$ denotes an averaging over the wave function $\varphi(\mathbf{r})[\Phi(\mathbf{R})]$.

Two different functional forms for the function $\varphi(\mathbf{r})$, referring to the relative motion, were chosen: (a) Coulombic type,

$$
\varphi(\mathbf{r})=\left(\frac{b}{2}\right]^{\beta+3 / 2}[4 \pi \Gamma(2 \beta+3)]^{-1 / 2} r^{\beta} e^{-b r / 4},
$$

and (b) oscillator type,

$$
\varphi(\mathbf{r})=\left(\frac{\Omega}{2}\right)^{\beta / 2+3 / 4}\left[2 \pi \Gamma\left(\beta+\frac{3}{2}\right)\right]^{-1 / 2} r^{\beta} e^{-\Omega r^{2} / 4},
$$

where $b, \Omega$, and $\beta$ are variational parameters.

For the center-of-mass motion, described by $\Phi(\mathbf{R})$, we tried wave functions of (a) oscillator type,

$$
\Phi(\mathbf{R})=\left(\frac{2 \Omega_{1}}{\pi}\right)^{3 / 4} e^{-\Omega_{1} R^{2}},
$$

and (b) of Pekar type,

$$
\Phi(\mathbf{R})=\frac{\gamma^{3 / 2}}{(7 \pi)^{1 / 2}}(1+\gamma R) e^{-\gamma R},
$$

where $\Omega_{1}$ and $\gamma$ are the variational parameters.

It is well known that such averaging leads to the correct power-law behavior of the energy $E \propto-\alpha^{2}$ in the strong-coupling limit, but fails in the weak-coupling regime where it is known that the correct dependence is a linear one. Analogous procedures for the single-polaron energy $E_{1}$ result in

$$
E_{1}=-\frac{1}{2}\left\langle\left\langle\mathbf{P}^{2}\right\rangle\right\rangle-\sum_{\mathbf{k}}\left|\mathcal{A}_{\mathbf{k}}\right|^{2},
$$

where $\mathcal{A}_{\mathbf{k}}=V_{\mathbf{k}}\left\langle\left\langle e^{i \mathbf{k} \cdot \mathbf{R}}\right\rangle\right\rangle$. Using the wave functions (11), one obtains from Eq. (12), for the oscillator wave function,

$$
E_{1}=-\frac{\alpha^{2}}{3 \pi}=-0.106103 \alpha^{2},
$$

and for the Pekar-type wave function,

$$
E_{1}=-\frac{3^{5} 199^{2}}{2^{18} 7^{3}} \alpha^{2}=-0.107023 \alpha^{2} .
$$

Thus the above wave functions reproduce the leading term in the strong-coupling expansion. From previous studies we know that bipolarons can only exist above rather high critical values $\alpha_{c}$ of the electron-phonon coupling constant, e.g., $\alpha_{c}=7.3$ as found by Adamowski ${ }^{10}$ and $\alpha_{c}=6.9$ found by Verbist, Peeters, and Devreese. ${ }^{12}$ Consequently, the strong-coupling results are a valid first approximation. In the next section these results will be improved and the validity range will be extended to lower values of $\alpha$.

From Eqs. (10) and (11) we have four different variational estimates for the bipolaron energy. The condition under which a bipolaron can exist as a stable state is given by $E \leq 2 E_{1}$; i.e., the bipolaron energy must be lower than twice the single-polaron energy. This leads to a critical value $\eta_{c}$, where $\eta=\epsilon_{\infty} / \epsilon_{0}$ is the ratio of the dielectric constants. The corresponding critical value $U_{c}$ of the Coulomb repulsion strength, for a given $\alpha$, can be deduced from $U=\sqrt{2} \alpha /(1-\eta)$. Note that there is no critical value of $\alpha$ in this scheme, since in the strongcoupling expansion the energies $E$ and $E_{1}$ are proportional to $\alpha^{2}$. Our numerical results for $\eta_{c}$ are presented in Table I. In order to estimate critical values of $\eta$, one has to adopt a particular expression for the single polaron, which is not exactly known. Two different points of view are possible: (a) Take the best single-polaron energy available from literature. This route was followed by Adamowski ${ }^{10}$ who took the most general quadratic Feynman approximation. ${ }^{15}$ In the strong-coupling limit, 
TABLE I. Estimated critical values $\eta_{c}$ of the ratio of dielectric constants are presented for the different trial wave functions $\Psi(\mathbf{R}, \mathbf{r})=\Phi(\mathbf{R}) \varphi(\mathbf{r})$. These numbers result from a comparison between the bipolaron energy calculated with $\Psi(\mathbf{R}, \mathbf{r})$ and a single-polaron energy calculated with $\Phi(\mathbf{R})$. Lower bounds to the exact value of $\eta_{c}$ are given between parentheses. They were calculated through a comparison with the exact Miyake result as discussed in the text.

\begin{tabular}{cccccc}
\hline \hline Type & $\Phi(\mathbf{R})$ & $\varphi(\mathbf{r})$ & $\beta=0$ & $\beta=1$ & $\beta=2$ \\
\hline Ia & Oscillator & Coulomb & & 0.084 & 0.119 \\
& & & & $(0.072)$ & $(0.106)$ \\
Ib & Oscillator & Oscillator & 0.079 & 0.131 & 0.082 \\
& & & $(0.067)$ & $(0.119)$ & $(0.065)$ \\
IIa & Pekar & Coulomb & & 0.058 & 0.092 \\
& & & & $(0.050)$ & $(0.084)$ \\
IIb & Pekar & Oscillator & 0.052 & 0.104 & 0.061 \\
& & & $(0.045)$ & $(0.096)$ & $(0.051)$ \\
\hline \hline
\end{tabular}

studied above, an exact expression (to leading order in $\alpha$ ) for $E_{1}$ has been given by Miyake. ${ }^{16}$ Because the obtained bipolaron energy is an upper bound to the exact result, this procedure will give us a lower bound on $\eta_{c}$. In Table I this value has been given in brackets. (b) One can take the single-polaron energy, as is obtained by making similar approximations as for the bipolaron problem. [In the calculations made above, this corresponds to Eqs. (11) and (12).] The underlying idea is that, if one improves the approximation, both the bipolaron and single-polaron energies are expected to decrease. Consequently, $\eta_{c}$ is not expected to be altered substantially. In this approach, however, one has no criterion whether the obtained value of $\eta_{c}$ is an upper or lower bound to the exact value; in fact, both cases are possible. This point is made clearer in Fig. 1. We have plotted the exact bipolaron energy and (twice) the exact single-polaron energy as solid curves as a function of $\eta$; the dashed lines are upper bounds to these energies. Bipolaron formation is energetically favorable if $\eta \leq \eta_{c}$, where $\eta_{c}$ is the exact critical value. If $\eta_{c}^{\prime}$ and $\widetilde{\eta}_{c}^{\prime}$ are estimations of $\eta_{c}$ based on the upper bound to the bipolaron energy by comparing it to twice the exact (respectively, approximated) singlepolaron energy, it is seen that $\eta_{c}^{\prime}$ is a lower bound to $\eta_{c}$, while $\widetilde{\eta}_{c}^{\prime}$ is larger than $\eta_{c}$. It is not difficult to shift the approximate curves in such a way that $\widetilde{\eta}_{c}^{\prime}<\eta_{c}$; it is, however, impossible to realize a situation such that $\eta_{c}^{\prime}>\eta_{c}$ since the approximate bipolaron energy is an upper bound. The value of $\widetilde{\eta}_{c}^{\prime}$ obtained in this way is listed in Table I, and the lower bounds $\eta_{c}^{\prime}$ to the exact value $\eta_{c}$ are given between brackets.

The oscillator-oscillator wave function [case $\mathrm{Ib}(\beta=1)$ ] leads to the best approximation, and the maximal values for both $\widetilde{\eta}_{c}^{\prime}$ and the lower bound $\eta_{c}^{\prime}$ are obtained. A description of the relative motion with a Coulomb wave function leads to about $10 \%$ worse results [see case Ia $(\beta=2)$ in Table I]. The center-of-mass motion is best described by an oscillator wave function (cases I). Such a wave function can also be obtained as the strong-coupling limit of the path-integral treatment, which we will propose in the next section. The results in Table I will be further elaborated upon in Sec. IV, where a comparison with the available results in the literature is made.

Recently, ${ }^{12,14}$ it was shown that the bipolaron stability region is enlarged in two dimensions (2D) and that this result might be of importance for the high- $T_{c}$ superconductors. ${ }^{17}$ Therefore, we have repeated our calculations in $2 \mathrm{D}$ for the $\mathrm{Ib}$ case, which gives the highest lower bound to $\eta_{c}$ in $3 \mathrm{D}$. The results are given in Table II. As expected on the basis of scaling arguments, ${ }^{12}$ the $\beta=0$ result is the same in $3 \mathrm{D}$ as in $2 \mathrm{D}$. This is not true for the $\beta=0$ lower bound to $\eta_{c}$ since the exact Miyake result for the single-polaron energy does not scale. Perhaps more surprising are the $\beta=1$ results, which show an appreciable increase in the estimations for $\eta_{c}$.

\section{BIPOLARON SPIN-SINGLET STATE}

In the present section we will combine the best estimate for the relative motion, as obtained in the previous section, with a Feynman path-integral approximation to the center-of-mass motion.

As a first step, we apply the canonical transformation of Lee, Low, and Pines ${ }^{18}$ to the Hamiltonian (5), which

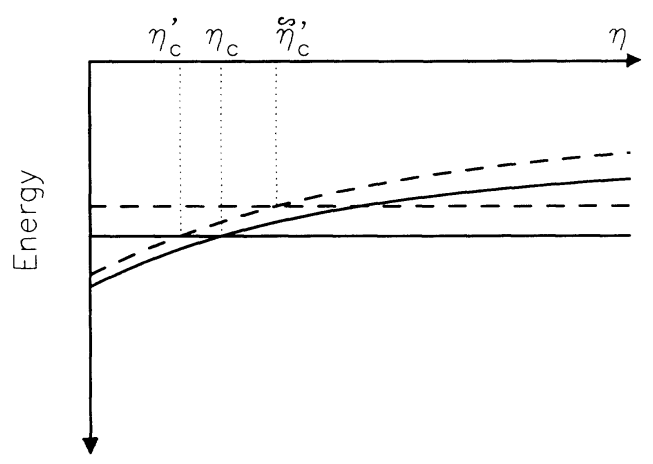

FIG. 1. Estimation of the critical value $\eta_{c}$ is sketched. The exact bipolaron energy is presented as a solid line; the horizontal solid line is (twice) the exact single-polaron energy. The dashed lines represent analogous approximate results. Estimating the critical value from a comparison between the approximate bipolaron as well as single-polaron energies results in a value $\widetilde{\eta}_{c}^{\prime}$ which is larger than $\eta_{c}$ in this situation. If the approximate bipolaron energy is compared to the exact single-polaron energy, we find a lower bound $\eta_{c}^{\prime}$ to the exact value $\eta_{c}$. 
TABLE II. For the best trial function in 3D, i.e., the oscillator-oscillator wave function $\mathrm{Ib}$, the calculations are repeated in $2 \mathrm{D}$ and the analog of Table $\mathrm{I}$ is constructed.

\begin{tabular}{cccc}
\hline \hline & $\beta=0$ & $\beta=1$ & $\beta=2$ \\
\hline 3D & 0.079 & 0.131 & 0.092 \\
& $(0.067)$ & $(0.119)$ & $(0.076)$ \\
2D & 0.079 & 0.158 & 0.013 \\
& $(0.063)$ & $(0.136)$ & \\
\hline \hline
\end{tabular}

results in ${ }^{6}$

$$
\begin{aligned}
H= & \frac{1}{4}\left(\mathbf{P}-\sum_{\mathbf{k}} \mathbf{k} a_{\mathbf{k}}^{\dagger} a_{\mathbf{k}}\right]^{2}+\mathbf{p}^{2} \\
& +2 \sum_{\mathbf{k}} \cos \left[\frac{\mathbf{k} \cdot \mathbf{r}}{2}\right]\left(V_{\mathbf{k}} a_{\mathbf{k}}+V_{\mathbf{k}}^{*} a_{\mathbf{k}}^{\dagger}\right)+\sum_{\mathbf{k}} a_{\mathbf{k}}^{\dagger} a_{\mathbf{k}}+\frac{U}{r} .
\end{aligned}
$$

Since the Hamiltonian is translational invariant, the total momentum $\mathbf{P}$ can be considered as a $c$ number.

The next step is to average the Hamiltonian (14) over the best trial wave function of the previous section. This function is symmetric under a permutation of the electrons $[\varphi(-\mathbf{r})=\varphi(\mathbf{r})]$, which implies that we are estimating the characteristics of the bipolaron spin-singlet state. In performing this average, we obtain the effective Hamiltonian

$$
\begin{aligned}
H= & \frac{1}{4}\left[\mathbf{P}-\sum_{\mathbf{k}} \mathbf{k} a_{\mathbf{k}}^{\dagger} a_{\mathbf{k}}\right]^{2}+\sum_{\mathbf{k}}\left(\mathfrak{B}_{\mathbf{k}} a_{\mathbf{k}}+\mathfrak{B}_{\mathbf{k}}^{*} a_{\mathbf{k}}^{\dagger}\right) \\
& +\sum_{\mathbf{k}} a_{\mathbf{k}}^{\dagger} a_{\mathbf{k}}+E_{r},
\end{aligned}
$$

where $\mathfrak{B}_{\mathbf{k}}=2 V_{\mathbf{k}}\langle\cos [(\mathbf{k} \cdot \mathbf{r}) / 2]\rangle$ is the renormalized electron-phonon coupling coefficient and $E_{r}$ is the energy related to the relative motion, as defined in Eq. (9).

The Hamiltonian (15) is in essence a single-polaron Hamiltonian. The differences with the usual Fröhlich Hamiltonian in single-polaron physics are the following: (a) The electron mass is doubled since the bipolaron contains two electrons, (b) the energy is shifted by $E_{r}$, and (c) the electron-phonon interaction coefficients are renormalized because we have averaged the internal motion of the bipolaron. Keeping in mind that the best estimate of Sec. II was obtained with an oscillator-type wave function $\Phi(\mathbf{R})$, we treat Hamiltonian (15) within the scope of the path-integral method. The advantage of this approach compared to the approach of Sec. II is that the energy will be lowered for intermediate values of the coupling constant $\alpha$. Furthermore, this scheme takes into account the translation invariance of the system and allows us to calculate the bipolaron-spin-singlet effective mass. The Feynman approximation, ${ }^{19}$ applied to Hamiltonian (15), results in the following upper bound to the ground-state energy of the bipolaron (units are such that $\left.\hbar=m=\omega_{\mathrm{LO}}=1\right)$ :

$E=\frac{3}{4} \frac{(v-w)^{2}}{v}-\sum_{\mathbf{k}}\left|\mathfrak{B}_{\mathbf{k}}\right|^{2} \int_{0}^{\infty} d s e^{-s-k^{2} F(s) / 4 v^{2}}+E_{r}$, and the effective mass is given by

$$
m^{*}=2+\frac{1}{3} \sum_{\mathbf{k}} \mathbf{k}^{2}\left|\mathfrak{B}_{\mathbf{k}}\right|^{2} \int_{0}^{\infty} d s s^{2} e^{-s-k^{2} F(s) / 4 v^{2}},
$$

where $v$ and $w$ are the usual Feynman variational parameters and $F(s)$ denotes the function

$$
F(s)=w^{2} s+\frac{v^{2}-w^{2}}{v}\left(1-e^{-v s}\right) .
$$

The rms separation between the electrons,

$$
r_{12}=\left[\left\langle\left(\mathbf{r}_{1}-\mathbf{r}_{2}\right)^{2}\right\rangle\right]^{1 / 2},
$$

is independent of the center-of-mass motion, i.e., of $v$ and $w$. Consequently, Eq. (9) can be applied to calculate $r_{12}$.

The expression for the average number $N$ of (virtual) phonons in the cloud around the electron can be derived as $^{20}$

$$
N=\left(1-\frac{3}{2} \alpha \frac{\partial}{\partial \alpha}-\frac{1}{2} U \frac{\partial}{\partial U}\right) E
$$

which gives the following result for the case under study:

$$
\begin{aligned}
N= & \frac{3}{4} \frac{(v-w)^{2}}{v}+E_{r}-\frac{1}{2} U\left\langle\frac{1}{r}\right\rangle \\
& -\frac{1}{2} \sum_{\mathbf{k}}\left|\mathfrak{B}_{\mathbf{k}}\right|^{2} \int_{0}^{\infty} d s e^{-s-k^{2} F(s) / 4 v^{2}} .
\end{aligned}
$$

In accordance with the results of Sec. II, we choose as a trial wave function $\varphi(\mathbf{r})$ the expression given by Eq. (10b) for $\beta=1$. With this choice we readily obtain

$$
\begin{aligned}
\frac{\mathfrak{B}_{\mathbf{k}}}{2 V_{\mathbf{k}}} & =\left\langle\cos \left(\frac{\mathbf{k} \cdot \mathbf{r}}{2}\right)\right\rangle \\
& =\left(1-\frac{k^{2}}{12 \Omega}\right) e^{-k^{2} / 8 \Omega}, \\
\left\langle\mathbf{p}^{2}\right\rangle & =\frac{7}{12} \Omega, \\
\left\langle\frac{1}{r}\right\rangle & =\frac{4}{3}\left[\frac{\Omega}{2 \pi}\right)^{1 / 2}, \\
\left\langle r^{2}\right\rangle & =\frac{5}{\Omega} .
\end{aligned}
$$

Inserting these results in Eq. (16), we obtain, for the bipolaron energy,

$$
\begin{aligned}
E=\frac{3}{4} \frac{(v-w)^{2}}{v}+\frac{7}{12} \Omega & +\frac{4}{3}\left[\frac{\Omega}{2 \pi}\right)^{1 / 2} U \\
-4\left(\frac{2 \Omega}{\pi}\right)^{1 / 2} \alpha \int_{0}^{\infty} d s e^{-s} \sqrt{f(s)} & \\
& \times\left(1-\frac{1}{3} f(s)+\frac{1}{12}[f(s)]^{2}\right),
\end{aligned}
$$

where we introduced $f(s)=\left[1+\left(\Omega / v^{2}\right) F(s)\right]^{-1}$ and $F(s)$ is given by Eq. (18). A numerical minimalization over the 
variational parameters $v, w$, and $\Omega$ gives an upper bound to the ground-state energy of the bipolaron spin-singlet state. With the estimated values of these variational parameters, we can calculate bipolaron characteristics such as the average number of phonons $N$, rms separation $r_{12}$, and effective mass $m^{*}$, which can be explicitly calculated as

$$
\begin{aligned}
& N=\frac{3}{4} \frac{(v-w)^{2}}{v}+\frac{7}{2} \Omega+\frac{2}{3} U\left(\frac{\Omega}{2 \pi}\right)^{1 / 2} \\
& +2\left(\frac{2 \Omega}{\pi}\right)^{1 / 2} \alpha \int_{0}^{\infty} d s e^{-s} \sqrt{f(s)} \\
& \times\left\{1-\frac{1}{3} f(s)+\frac{1}{12}[f(s)]^{2}\right\}, \\
& r_{12}=\left(\frac{5}{\Omega}\right]^{1 / 2} \\
& m^{*}=2+\frac{8}{3} \Omega\left(\frac{2 \Omega}{\pi}\right]^{1 / 2} \alpha \int_{0}^{\infty} d s s^{2} e^{-s}[f(s)]^{3 / 2} \\
& \times\left\{1-f(s)+\frac{5}{12}[f(s)]^{2}\right\} .
\end{aligned}
$$

In Figs. 2 and 3 we compare the present results (solid line) for the energy [Eq. (23)] with the path-integral calculation of Ref. 11 (dashed line), which utilizes a full path-integral treatment also for the relative coordinate. Figure 2 shows the energy $E$ as function of the repulsion $U$ at fixed electron-phonon coupling constant $\alpha=10$. The full path-integral calculation gives a lower value for the energy throughout the whole range. Thus the stability region for bipolaron formation is much smaller in the present model. From Sec. II, however, it is concluded that the present model $\left(\eta_{c}=1.31\right)$ should give a larger stability region as $\alpha$ increases compared with the model

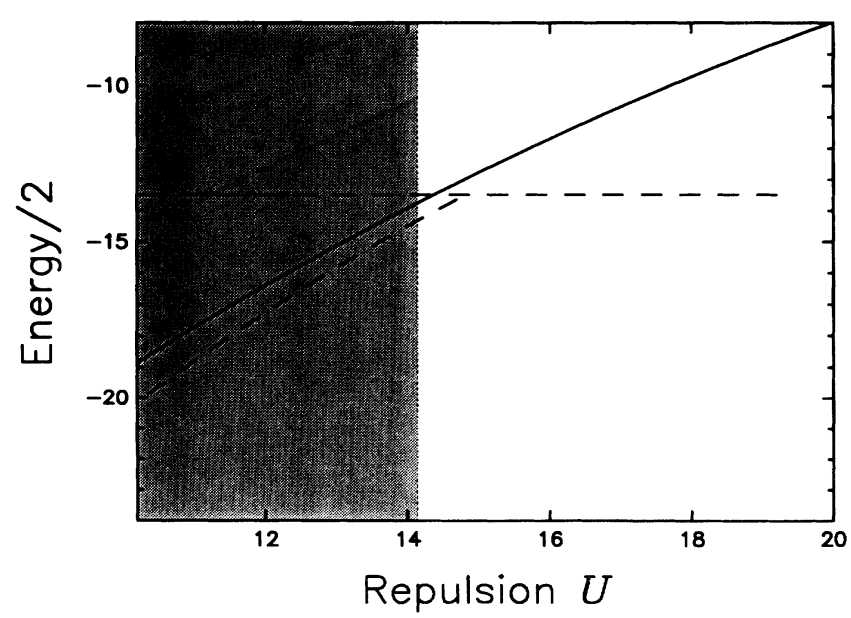

FIG. 2. Bipolaron energy $E$ is plotted as a function of the repulsion strength $U$ for fixed coupling constant $\alpha=10$. The solid line presents the result of this model; the dashed line is the result of the full path-integral treatment (Ref. 12). (The shaded area represents the "unphysical" region $U<\sqrt{2} \alpha$.)

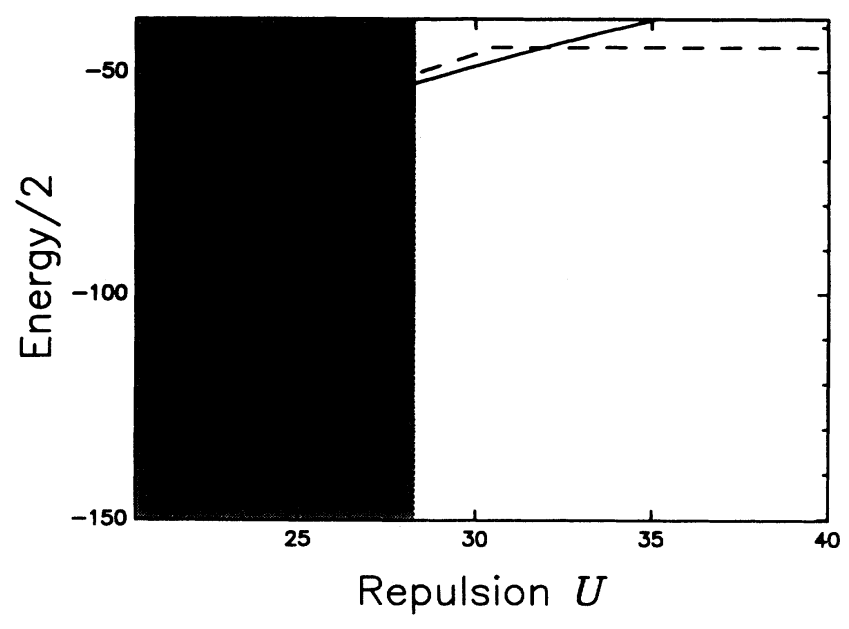

FIG. 3. Bipolaron energy $E$ is plotted as a function of the repulsion strength $U$ for fixed coupling constant $\alpha=20$. The solid line presents the result of this model; the dashed line is the result of full path-integral treatment (Ref. 12). In comparison to Fig. 1, the stability region for bipolaron formation is much enhanced for this larger value of $\alpha$. (The shaded area represents the "unphysical" region $U<\sqrt{2} \alpha$.)

of Ref. 11, from which we obtain $\eta_{c}=0.079$. In Fig. 3 this is demonstrated for $\alpha=20$, where the energy $E$ is plotted as function of the repulsion $U$. Here it is found that the stability region of the present model is roughly one and half times larger than for the model of Ref. 11. In Fig. 4 we compare the phase diagram of both models. The shaded area represents the stability region of the present model. The region $U<\sqrt{2} \alpha$ is unphysical since it would imply a negative static dielectric constant $\epsilon_{0}$. The stability region calculated in Ref. 11 lies below the thick dashed line. The present model has a critical coupling constant $\alpha_{c}=9.3$, above which bipolarons can exist. This value is larger than the one found in Ref. 11. For completeness, the asymptotic estimations $U=\sqrt{2} \alpha$ / $\left(1-\eta_{c)}\right.$ are indicated as thin lines (solid line, present model; dashed line, Ref. 11). In Figs. 5-7 the number of phonons $N$, rms separation $r_{12}$, and effective mass $m^{*}$ are plotted as a function of the repulsion $U$ for $\alpha=20$; the solid line indicates the present calculation, while the dashed line is taken from Ref. 11.

From the strong-coupling analysis in Sec. II, one expects a more pronounced effect if the present model is applied in 2D. Therefore, we have repeated our calculations in 2D. For completeness, the formulas for the energy $E$, number of phonons $N$, rms separation $r_{12}$, and effective mass $m^{*}$ are presented:

$$
\begin{aligned}
& E=\frac{1}{2} \frac{(v-w)^{2}}{v}+\frac{1}{2} \Omega+\frac{1}{4}\left(\frac{\pi \Omega}{2}\right)^{1 / 2} U \\
& -2\left(\frac{2 \Omega}{\pi}\right)^{1 / 2} \alpha \int_{0}^{\infty} d s e^{-s} \sqrt{f(s)} \\
& \times\left\{1-\frac{1}{2} f(s)+\frac{3}{16}[f(s)]^{2}\right\},
\end{aligned}
$$




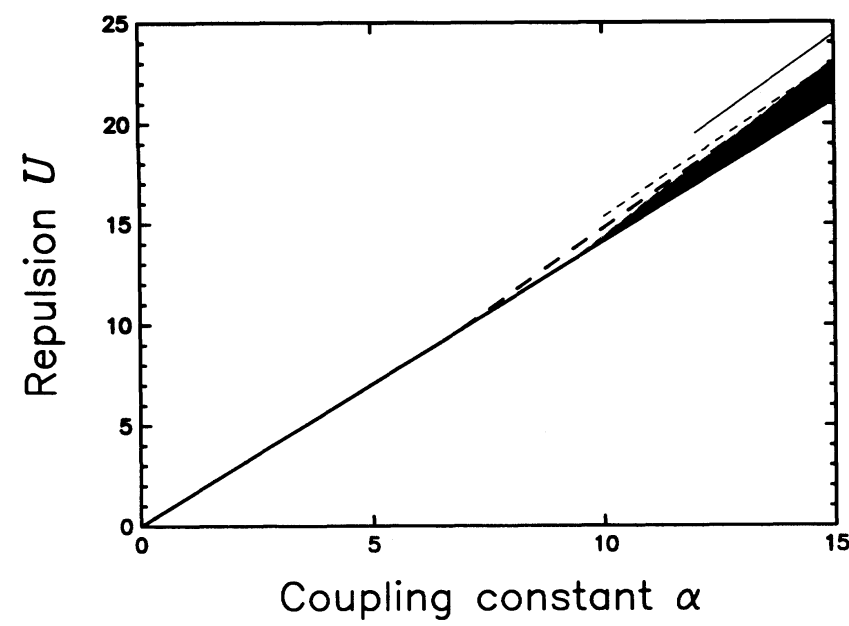

FIG. 4. Phase diagram for bipolaron formation in 3D is presented. The shaded area represents the stability region for bipolaron formation as obtained with the present model. The thick solid line is $U=\sqrt{2} \alpha$, above which the physical acceptable values $(\alpha, U)$ are situated. The stability region, obtained in Ref. 9, lies below the thick dashed line. Asymptotic results are indicated as thin lines [solid line, present calculation $(\eta=0.131)$; dashed line, Refs. 12 and $13(\eta=0.079)]$. The critical value of the electron-phonon constant above which bipolaron formation is possible is $\alpha_{c} \approx 9.3$.

$$
\begin{aligned}
& N=\frac{1}{2} \frac{(v-w)^{2}}{v}+\frac{1}{2} \Omega \\
&+\left(\frac{2 \Omega}{\pi}\right)^{1 / 2} \alpha \int_{0}^{\infty} d s e^{-s \sqrt{f(s)}} \\
& \\
& \times\left\{1-\frac{\pi}{2} f(s)+\frac{3}{16}[f(s)]^{2}\right\},
\end{aligned}
$$

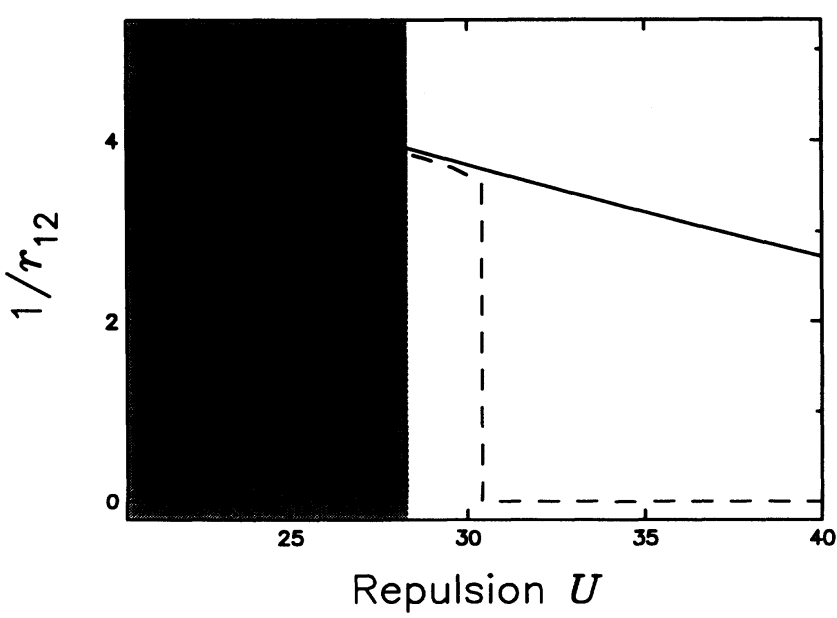

FIG. 5. At fixed coupling $\alpha=20$, the rms separation $r_{12}$ is plotted as function of the repulsion $U$. The solid line represents the model of Sec. II, while the dashed line stems from Ref. 12. (The shaded area represents the "unphysical" region $U<\sqrt{2} \alpha$.)

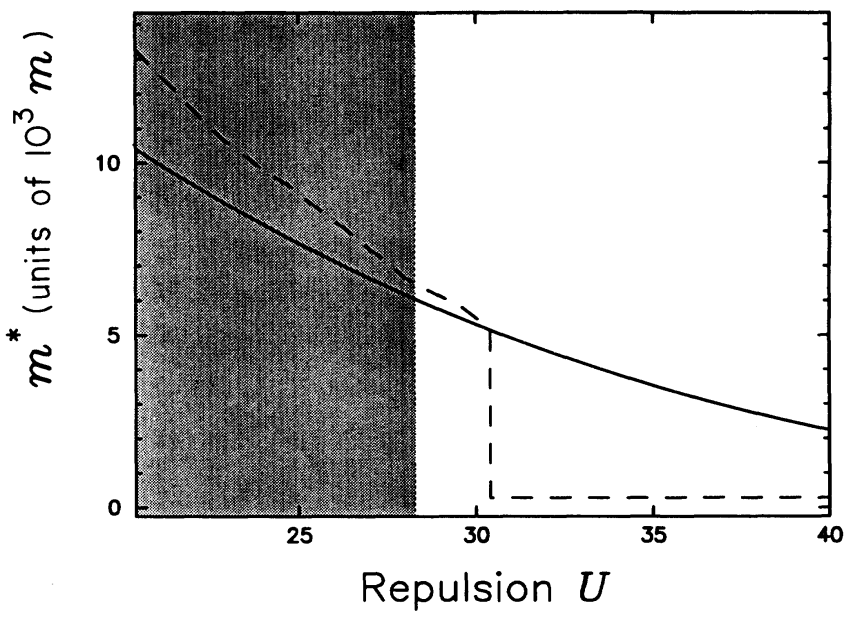

FIG. 6. At fixed coupling $\alpha=20$, the effective bipolaron mass $m^{*}$ is plotted as function of the repulsion $U$. The solid line represents the model of Sec. II, while the dashed line stems from Ref. 12. (The shaded area represents the "unphysical" region $U<\sqrt{2} \alpha$.)

$$
\begin{aligned}
& r_{12}=\left(\frac{2}{\Omega}\right]^{1 / 2}, \\
& m^{*}=2+2 \Omega\left[\frac{2 \Omega}{\pi}\right]^{1 / 2} \alpha \int_{0}^{\infty} d s s^{2} e^{-s}[f(s)]^{3 / 2} \\
& \times\left\{1-\frac{3}{2} f(s)+\frac{15}{16}[f(s)]^{2}\right\} .
\end{aligned}
$$

In Fig. 8 the 2D phase diagram is plotted. As expected from the asymptotic analysis, the stability region (shaded area) is much larger for large values of $\alpha$ than the stability region of the full path-integral treatment (dashed line).

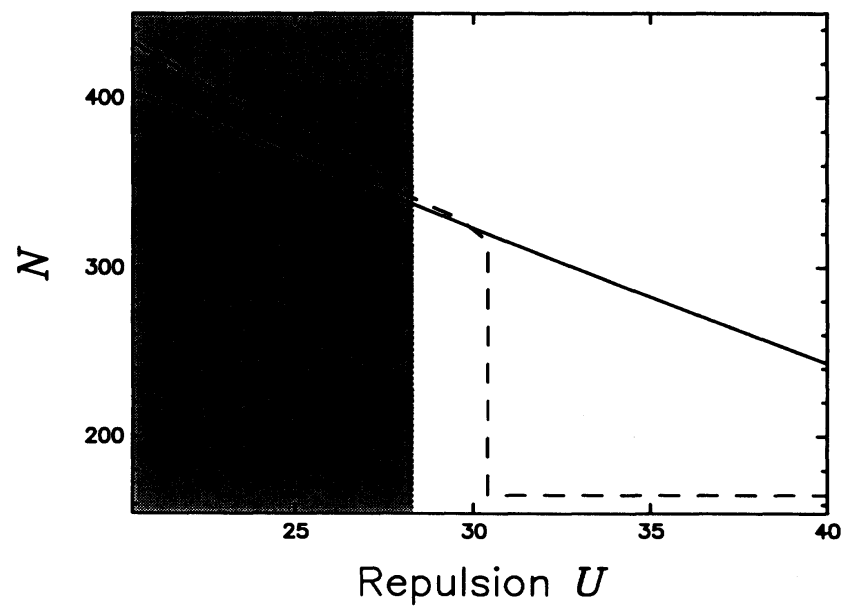

FIG. 7. At fixed coupling $\alpha=20$, the number of (virtual) phonons in the electron cloud, $N$, is plotted as function of the repulsion $U$. The solid line represents the model of Sec. II, while the dashed line stems from Ref. 12. (The shaded area represents the "unphysical" region $U<\sqrt{2} \alpha$.) 


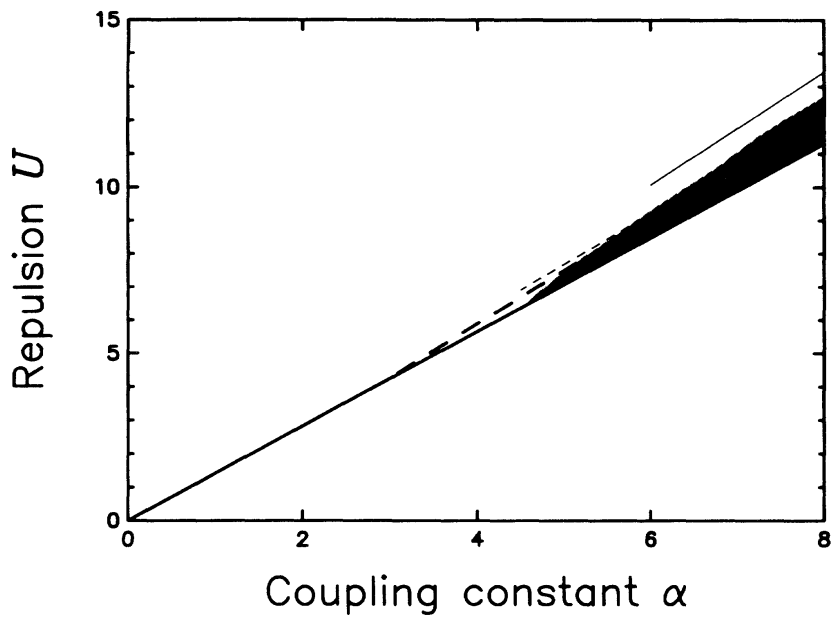

FIG. 8. Phase diagram for bipolaron formation in 2D is presented. The shaded area represents the stability region for bipolaron formation as obtained with the present model. The thick solid line is $U=\sqrt{2} \alpha$, above which the physical acceptable values $(\alpha, U)$ are situated. The stability region, obtained in Ref. 12 , lies below the thick dashed line. Asymptotic results are indicated as thin lines [solid line, present calculation $(\eta=0.131)$; dashed line, Refs. 12 and $13(\eta=0.079)]$. The critical value of the electron-phonon constant above which bipolaron formation is possible is $\alpha_{c} \approx 4.5$.

The thick solid line again represents $U=\sqrt{2} \alpha$. Similar to the $3 \mathrm{D}$ phase diagram, we have indicated the asymptotic results by thin lines. In $2 \mathrm{D}$ the present model leads to a critical coupling constant $\alpha_{c} \approx 4$.5. This value must be compared with $\alpha_{c} \approx 2.8$, as cited in Ref. 12 .

\section{COMPARISON WITH LITERATURE AND DISCUSSION}

We begin with a discussion of the data presented in Table I. In reality, it reproduces all strong-coupling results for $\eta_{c}$ as found in the literature. Vinetsky ${ }^{5}$ averaged the initial Hamiltonian over the space wave function

$$
\Psi_{V}\left(\mathbf{r}_{1}, \mathbf{r}_{2}\right) \propto \varphi\left(\mathbf{r}_{1}\right) \varphi\left(\mathbf{r}_{2}\right),
$$

where

$$
\varphi(\mathbf{r}) \propto\left[1+a r+c(a r)^{2}\right] e^{-a r}
$$

(henceforth we will use the proportionality symbol $\propto$ to omit irrevelant normalization factors). $\varphi(\mathbf{r})$ is the improved Pekar-type wave function, which leads to the correct strong-coupling energy. His result $\left(\eta_{c}=0.05\right)$ is reproduced in Table I as the IIa $(\beta=0)$ case. A significant improvement of this result was obtained by Suprun and Moizhes ${ }^{8}$ who included the electron correlation by choosing the trial wave function

$$
\Psi_{\mathrm{SM}}\left(\mathbf{r}_{1}, \mathbf{r}_{2}\right) \propto \varphi\left(r_{1}\right) \varphi\left(r_{2}\right)\left(1+c\left|\mathbf{r}_{1}-\mathbf{r}_{2}\right|\right),
$$

where

$$
\varphi(\mathbf{r}) \propto[1+a r] e^{-a r} .
$$

Their result $\left(\eta_{c}=0.14\right)$ is almost reproduced in Table I through the Ia $(\beta=2)$ or $\mathrm{Ib}(\beta=1)$ cases. The result by
Suprun and Moizhes coincides with the strong-coupling limit of Adamowski. ${ }^{10}$ The techniques of the papers ${ }^{5,8,10}$ considered so far allow also for the calculation of the bipolaron radius, but not for the bipolaron mass. Nevertheless, the estimates of the critical values of $\eta=\epsilon_{\infty} / \epsilon_{0}$ by Suprun and Moizhes and Adamowski lead to the largest value, i.e., $\eta_{c}=0.14$.

Mitra $^{11}$ reported the result $\eta_{c}=\frac{7}{15} \approx 0.47$, which is out of the limits of any other result in Table I. As a starting point, Mitra used the Hamiltonian (14), which was derived by Kochetov et al. ${ }^{6}$ For the relative motion, Mitra used a trial wave function $\varphi(\mathbf{r}) \propto e^{-\lambda r}$, which is the wave function of our Ia $(\beta=0)$ case. As can be seen from Table I, we did not obtain any bound state at all. The origin of this discrepancy is a mistake in Mitra's calculation at its final stage. Mitra used a canonical transformation to shift the creation and annihilation operators,

$$
a_{\mathrm{k}} \rightarrow a_{\mathrm{k}}-\xi_{\mathrm{k}}, \quad a_{\mathrm{k}}^{\dagger} \rightarrow a_{\mathrm{k}}^{\dagger}-\xi_{\mathrm{k}}^{*},
$$

and consequently the vacuum average was obtained in the form

$$
E_{M}=2 \lambda^{2}-\alpha \lambda\left(5-\frac{2}{1-\eta}\right) .
$$

Minimization of Eq. (29) over $\lambda$ leads to

$$
E_{M}=-\frac{\alpha^{2}}{8}\left[5-\frac{2}{1-\eta}\right)^{2},
$$

which Mitra compared with $2 E_{1}$ by taking $E_{1}=-\alpha^{2}$ $(25 / 256)$. This is the single-polaron energy obtained with a Coulomb-type wave function. In calculating the vacuum averaging over the first term in Eq. (15), Mitra took this term equal to zero, which is not valid. After correcting this, Eq. (32) takes the form

$$
E_{M}^{\prime}=8 \alpha \lambda^{3}+2 \lambda^{2}-\lambda \alpha\left(5-\frac{2}{1-\eta}\right) \text {. }
$$

This corrected result does not lead to bipolaron formation, in accordance with our results in Table I.

In a recent paper by Bassani et al. ${ }^{14}$ the Hamiltonians (5) and (14) were used. These two cases were strictly distinguished by the authors. Dealing with Eq. (14), they introduced a rather complicated technique of an expansion in partial waves in correspondence to the different angular momentum values of the two-electron system. They neglected triple and quartic powers of $a_{\mathrm{k}}$ and $a_{\mathrm{k}}^{\dagger}$ in the first term of Eq. (14). Consequently, they averaged over a trial wave function $\varphi(\mathbf{r})$ of Coulombic type [Eq. (10a)]. A critical value $\eta_{c}=0.084$ was found. This coincides with the result of Table I for the Ia $(\beta=1)$ case. This coincidence, however, is accidental because the strong-coupling limit was not obtained.

Considering the Hamiltonian (5), Bassani et al. averaged over a trial wave functions $\Phi(\mathbf{R})$ and $\varphi(\mathbf{r})$, which are both of oscillator type. They obtained a result which is identical to our $\mathrm{Ib}(\beta=0)$ case. They stressed that there exists an important difference between the two parts of their paper: The Hamiltonian (5) would correspond to a 
free bipolaron, while the Hamiltonian (14) would describe a localized bipolaron (after averaging). In our opinion this distinction is not that crucial as far as the bipolaron energy is concerned. Indeed, the wave function $\Phi(\mathbf{R})$ describes mostly the vibration of an electron in a polaron well. In Sec. III we demonstrated how to obtain the strong-coupling results with Hamiltonian (14). Moreover, the case Ib $(\beta=0)$ can also be obtained from the Feynman technique, which conserves translational invariance explicitly. As such, the formula for the energy by Bassani et al. as derived from Eq. (5) coincides completely with those of Ref. 12. Here it was derived with Eq. (14).

\section{CONCLUSIONS}

An asymptotic analysis of the bipolaron problem for $\alpha \rightarrow \infty$ is presented. As a first step, a transformation is made to center-of-mass coordinates for the electrons. The phonons are treated with a displaced oscillator approximation. The electronic wave is supposed to be a product wave function. It is shown that, using a Pekartype or oscillator wave function for the center-of-mass coordinate and a Coulomb or oscillator wave function for the relative coordinate, all the results published in the literature can be recovered. The best estimate of the critical ratio $\eta_{c}$ of the dielectric constants, as well as the highest value of its lower bound, is obtained with a oscillator-type trial wave function for both the center-of- mass and relative motions. Surprisingly, the Pekar-type wave function, which is the most succesful in singlepolaron theory, is slightly worse than the oscillator wave function for the center of mass. The results are summarized in Table $I$.

Taking the best approximation in $3 \mathrm{D}$, we performed the equivalent calculations in $2 \mathrm{D}$. It is found that, asymptotically, the stability region for bipolaron formation is much larger in $2 \mathrm{D}$ as compared with $3 \mathrm{D}$.

In Sec. III a model was introduced which combines the averaging of the relative coordinate over the asymptotically best wave function with a path-integral treatment of the center-of-mass motion. This results in an increase of the stability region for bipolaron formation in both two and three dimensions compared with the full pathintegral treatment ${ }^{12}$ at large values of the coupling constant $\alpha$. The critical values of the coupling constant, derived with this model, are $\alpha_{c} \approx 9.3$ in $3 \mathrm{D}$ and $\alpha_{c} \approx 4.5$ in 2D. These values are larger than those found within the framework of a full path-integral treatment. Figures 4 and 5 show the phase diagrams obtained with the presented model.

\section{ACKNOWLEDGMENTS}

One of us (F.M.P.) is supported by the Belgian National Science Foundation. Financial support was provided by Grant No. I.U.A.P.-11 as well as Contract No. 4.0006.90 and No. 2.0093.91 of the N.F.W.O.
${ }^{*}$ Permanent address: The Joint Institute for Nuclear Research (Dubna), Head Post Office P.O. Box 79, Moscow, U.S.S.R.

†Also at: Universiteit Antwerpen (RUCA), Groenborgerlaan 171, 2020 Antwerpen, Belgium and Eindhoven University of Technology, Department of Physics, P.O. Box 513, 5600 MB Eindhoven, The Netherlands.

${ }^{1}$ D. Emin, Phys. Rev. Lett. 62, 1544 (1989); D. Emin and M. S. Hillery, Phys. Rev. B 39, 6575 (1989).

${ }^{2}$ M. R. Shafroth, Phys. Rev. 100, 463 (1955).

${ }^{3}$ R. Friedberg, T. D. Lee, and H. C. Ren, Ann. Phys. (N.Y.) 208, 149 (1991).

${ }^{4}$ V. A. Moskalenko, Utcheniye Zapiski (fiz.-mat.) (Kishinev State University, City, 1955), Vol. XVII, p. 103 (in Russian).

${ }^{5}$ V. L. Vinetskii, Zh. Eksp. Teor. Fiz. 40, 1459 (1961) [Sov. Phys. JETP 13, 1023 (1961)].

${ }^{6}$ E. A. Kochetov, S. P. Kuleshov, V. A. Matveev, and M. A. Smondyrev, Teor. Mat. Fiz. 30, 183 (1977) [Theor. Math. Phys. 30, 117 (1978)].

${ }^{7}$ Y. Takada, Phys. Rev. B 26, 1223 (1982).

${ }^{8}$ S. G. Suprun and B. Ya Moizhes, Fiz. Tverd. Tela 24, 1571 (1982) [Sov. Phys. Semicond. 16, 700 (1982)].

${ }^{9}$ H. Hiramoto and Y. Toyozawa, J. Phys. Soc. Jpn. 54, 245
(1985).

10J. Adamowski, Acta Phys. Pol. A 73, 345 (1988); Phys. Rev. B 39, 3649 (1989).

${ }^{11}$ T. K. Mitra, Phys. Lett. A 142, 398 (1989).

${ }^{12}$ G. Verbist, F. M. Peeters, and J. T. Devreese, Phys. Rev. B 43, 2712 (1991).

${ }^{13}$ E. A. Kochetov and M. A. Smondyrev, Teor. Mat. Fiz. 85, 74 (1990).

${ }^{14}$ F. Bassani, M. Geddo, G. Iadonisi, and D. Ninno, Phys. Rev. B 43, 5296 (1991).

15J. Adamowski, B. Gerlach, and H. Leschke, in Functional Integration - Theory and Applications, edited by J. P. Antoine and E. Tirapegui (Plenum, New York, 1980), p. 291.

${ }^{16}$ S. Miyake, J. Phys. Soc. Jpn. 38, 181 (1975).

${ }^{17}$ G. Verbist, F. M. Peeters, and J. T. Devreese, Solid State Commun. 76, 1005 (1990).

${ }^{18}$ T. D. Lee, F. Low, and D. Pines, Phys. Rev. 90, 297 (1953).

${ }^{19}$ R. P. Feynman, Phys. Rev. 97, 660 (1955); Statistical Mechanics (W.A. Benjamin, Reading, MA, 1972).

${ }^{20}$ M. A. Smondyrev, Teor. Mat. Fiz. 68, 29 (1986) [Theor. Math. Phys. 68, 653 (1987)]. 


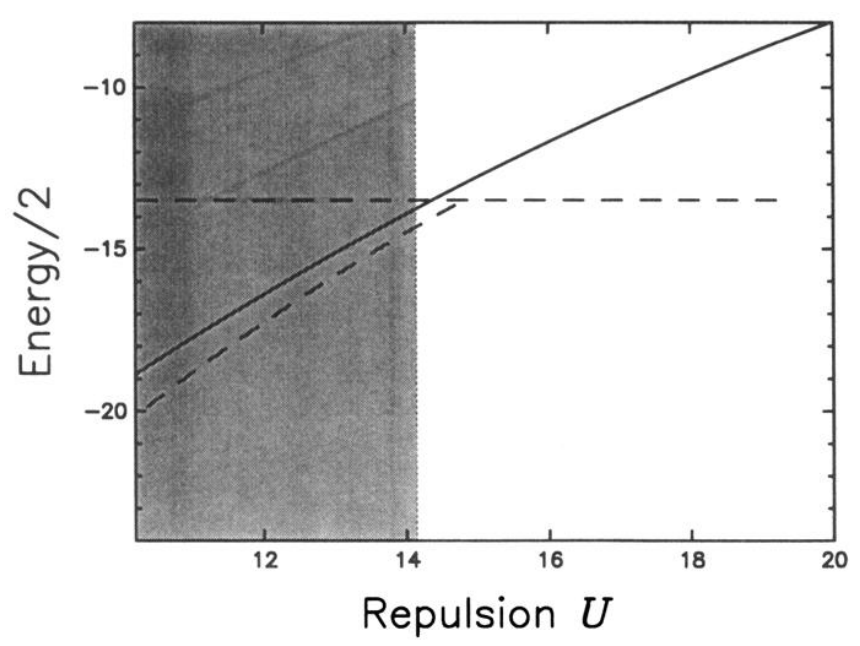

FIG. 2. Bipolaron energy $E$ is plotted as a function of the repulsion strength $U$ for fixed coupling constant $\alpha=10$. The solid line presents the result of this model; the dashed line is the result of the full path-integral treatment (Ref. 12). (The shaded area represents the "unphysical" region $U<\sqrt{2} \alpha$.) 


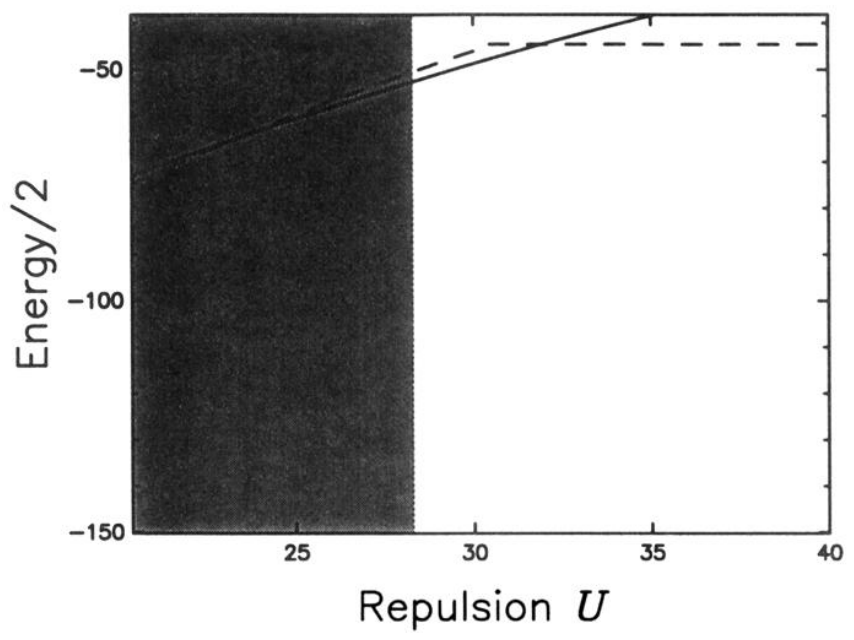

FIG. 3. Bipolaron energy $E$ is plotted as a function of the repulsion strength $U$ for fixed coupling constant $\alpha=20$. The solid line presents the result of this model; the dashed line is the result of full path-integral treatment (Ref. 12). In comparison to Fig. 1, the stability region for bipolaron formation is much enhanced for this larger value of $\alpha$. (The shaded area represents the "unphysical" region $U<\sqrt{2} \alpha$.) 


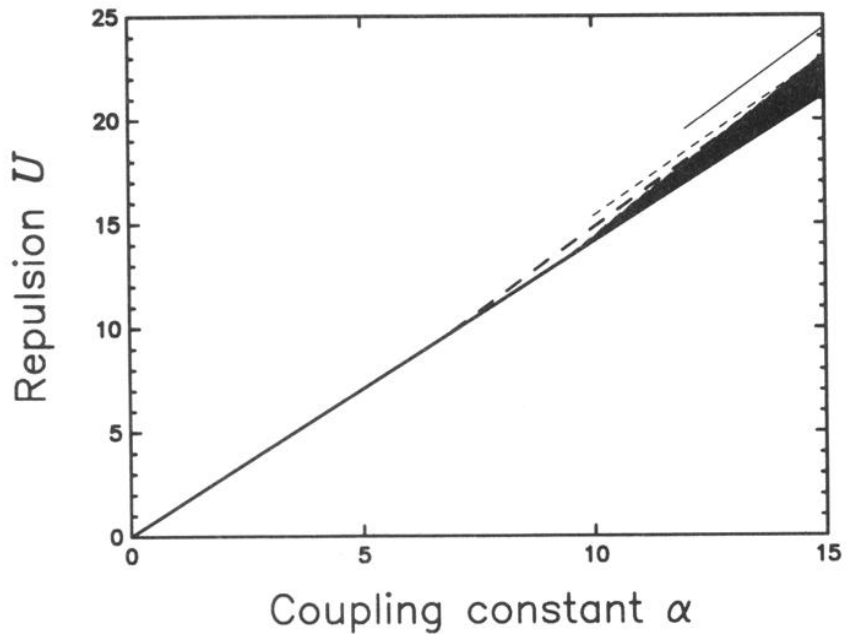

FIG. 4. Phase diagram for bipolaron formation in 3D is presented. The shaded area represents the stability region for bipolaron formation as obtained with the present model. The thick solid line is $U=\sqrt{2} \alpha$, above which the physical acceptable values $(\alpha, U)$ are situated. The stability region, obtained in Ref. 9, lies below the thick dashed line. Asymptotic results are indicated as thin lines [solid line, present calculation $(\eta=0.131)$; dashed line, Refs. 12 and $13(\eta=0.079)$ ]. The critical value of the electron-phonon constant above which bipolaron formation is possible is $\alpha_{c} \approx 9.3$. 


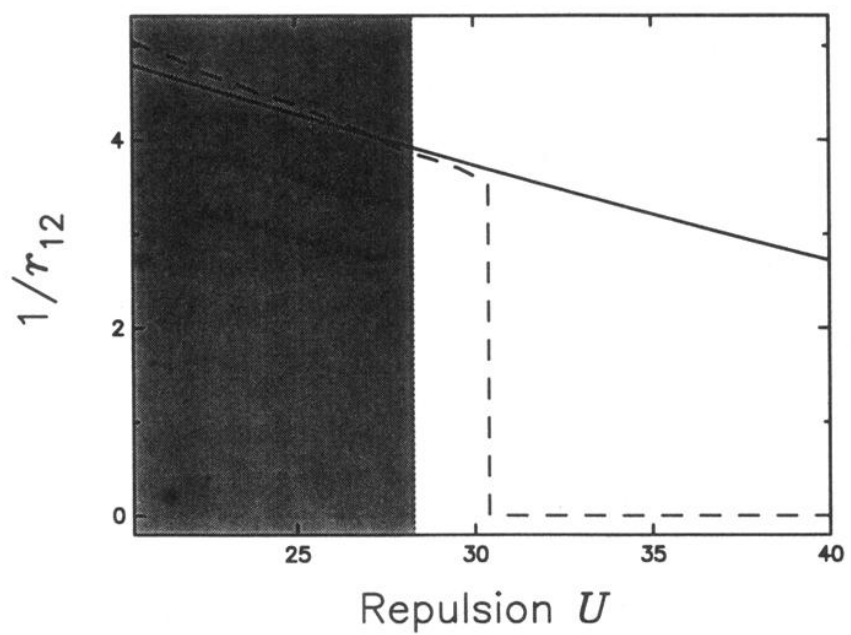

FIG. 5. At fixed coupling $\alpha=20$, the rms separation $r_{12}$ is plotted as function of the repulsion $U$. The solid line represents the model of Sec. II, while the dashed line stems from Ref. 12. (The shaded area represents the "unphysical" region $U<\sqrt{2} \alpha$.) 


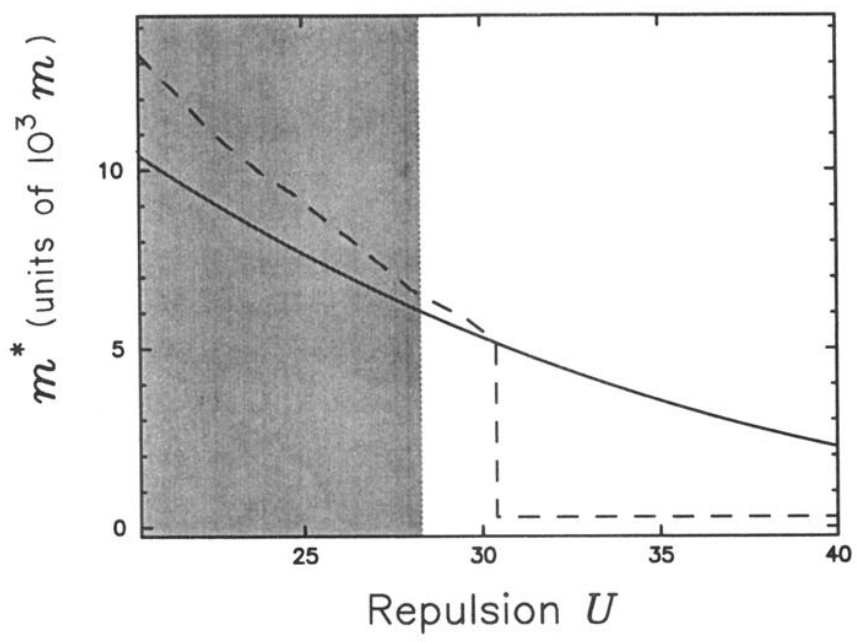

FIG. 6. At fixed coupling $\alpha=20$, the effective bipolaron mass $m^{*}$ is plotted as function of the repulsion $U$. The solid line represents the model of Sec. II, while the dashed line stems from Ref. 12. (The shaded area represents the "unphysical" region $U<\sqrt{2} \alpha$.) 


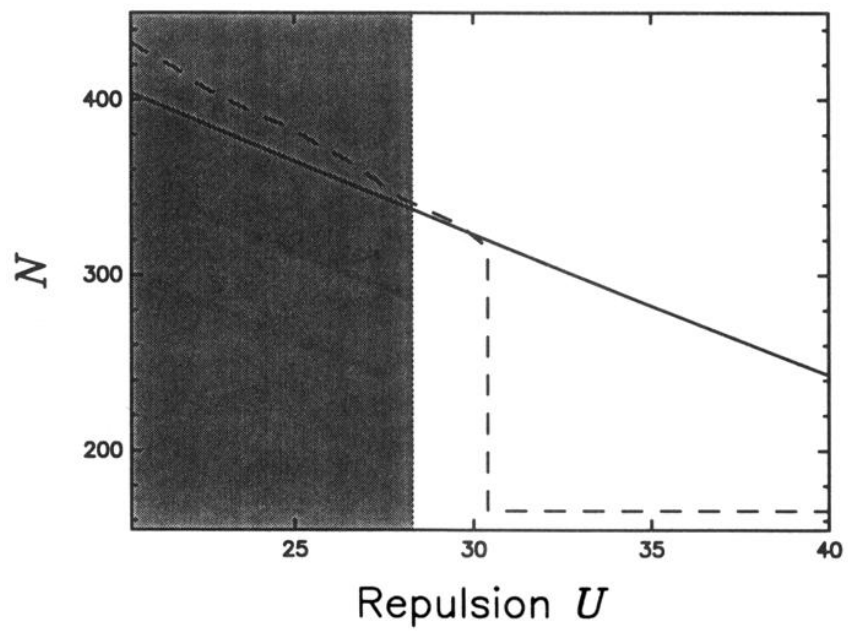

FIG. 7. At fixed coupling $\alpha=20$, the number of (virtual) phonons in the electron cloud, $N$, is plotted as function of the repulsion $U$. The solid line represents the model of Sec. II, while the dashed line stems from Ref. 12. (The shaded area represents the "unphysical" region $U<\sqrt{2} \alpha$.) 


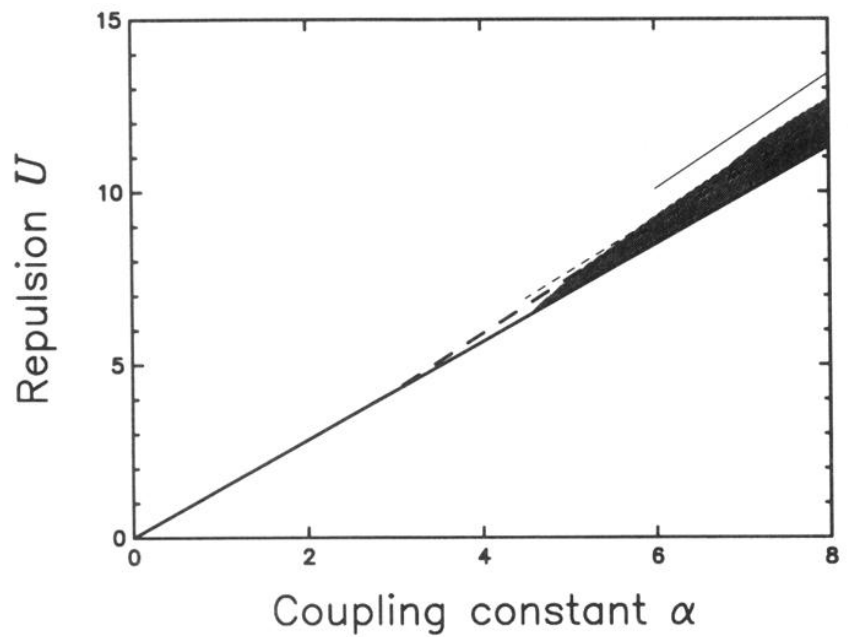

FIG. 8. Phase diagram for bipolaron formation in $2 \mathrm{D}$ is presented. The shaded area represents the stability region for bipolaron formation as obtained with the present model. The thick solid line is $U=\sqrt{2} \alpha$, above which the physical acceptable values $(\alpha, U)$ are situated. The stability region, obtained in Ref. 12 , lies below the thick dashed line. Asymptotic results are indicated as thin lines [solid line, present calculation $(\eta=0.131)$; dashed line, Refs. 12 and $13(\eta=0.079)$ ]. The critical value of the electron-phonon constant above which bipolaron formation is possible is $\alpha_{c} \approx 4.5$. 